\title{
"Atypical Cell" Parameter in Automated Urine Analysis for the Diagnosis of Bladder Cancer: A Retrospective Pilot Study
}

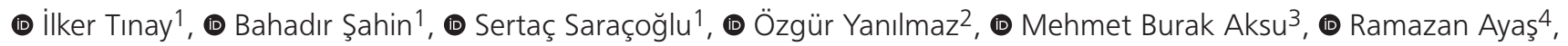 \\ (1) Deniz Filinte ${ }^{5}$, (1) Haydar Kamil Çam ${ }^{1}$, (1) Arzu Illki ${ }^{3}$ \\ ${ }^{1}$ Marmara University School of Medicine, Department of Urology, Istanbul, Turkey \\ 2 Marmara University School of Medicine, Department of Microbiology Laboratory, Istanbul, Turkey \\ 3 Marmara University School of Medicine, Department of Microbiology, Istanbul, Turkey \\ ${ }^{4}$ Sysmex Turkey Diagnostic Systems Department of Marketing, Istanbul, Turkey \\ ${ }_{5}$ Marmara University School of Medicine, Department of Pathology, Istanbul, Turkey
}

\begin{abstract}
Objective: To evaluate the application of an automated urine analyzer (AUA) for the diagnosis of bladder cancer (BC)

Materials and Methods: A retrospective data analysis of 2365 urine specimens from the department of urology has been performed and matched with those patients, who have undergone cystoscopic evaluation or surgical treatment for different urological pathologies during 2018. After matching, clinical records of the patients has been further evaluated in order to select patients with recent or previous BC diagnosis. To assess the diagnostic efficacy of AUA, patients were divided into five groups according to the patient history of BC and healthy controls.

Results: A total of 106 patients are included in this study and the majority (69.8\%) of the patients are follow-up patients with previous diagnosis and treatment of non-muscle invasive BC (NMIBC). For patients with low-risk NMIBC ( $=27)$, the sensitivity and specificity were calculated as $75 \%$ and $100 \%$. For patients with high-risk NMIBC $(n=47)$, who were previously treated with intravesical BCG, the sensitivity and specificity were calculated as $54.5 \%$ and $83.3 \%$. All patients in radical cystectomy group $(n=7)$ with muscle invasive $B C$ had positive urine analyses results for atypical cells. And none of the patients in the control group $(n=8)$ had positive AUA results and cystoscopic evaluation also did not show any bladder mass suspicious for BC.

Conclusion: The results of this retrospective pilot study showed acceptable sensitivity and specificity rates of the "fluorescence flow cytometry" based AUA and the results of the low-risk group are especially valuable regarding its potential use to decide on performing a follow-up cystoscopy or not. A prospective study is currently on progress to validate the findings of the current study.
\end{abstract}

Keywords: Bladder cancer, automated urine analysis, diagnosis

\section{Introduction}

Bladder cancer $(B C)$ is the $9^{\text {th }}$ most lethal malignancy with a worldwide age-standardized incidence rate (per 100,000 people/ years) of 9.0 for men and 2.2 for women (1). Cystoscopy, with a sensitivity of $90 \%$, is the golden standard for the diagnosis and follow-up of BC. However, it is an invasive approach that sometimes overlooks small tumors within the bladder (2). There are currently six Food and Drug Administration (FDA) approved urine tests, and although the sensitivity of these kits for $\mathrm{BC}$ diagnosis ranges from $57-82 \%$ to $74-88 \%$, it increases with increasing stage and degree of the $\mathrm{BC}$ (3). There is certainly a need for a non-invasive tool to replace surveillance cystoscopies. The fully automated urine formed element analyzer (UF-5000 SYSMEX Corporation, Kobe, JAPAN), which was launched in 2015, is capable of classifying and quantifying epithelial cells in greater detail with its "nucleic acid content" detecting

Cite this article as: Tınay I, Şahin B, Saraçoğlu S, Yanılmaz Ö, Aksu MB, Ayaş R, Filinte D, Çam HK, Illki A. "Atypical Cell" Parameter in Automated Urine Analysis for the Diagnosis of Bladder Cancer: A Retrospective Pilot Study. Bull Urooncol 2020;19(1):17-19

Address for Correspondence: İlker Tınay, Marmara University Faculty of Medicine, Department of Urology, İstanbul, Turkey E-mail: ilker tinay@yahoo.com ORCID-ID: orcid.org/0000-0001-6768-9373 Received: 13.10.2019 Accepted: 25.12.2019 
"fluorescence flow cytometry" technology (4). Epithelial cells, predominantly with high nucleic acid contents for their cell size, are counted as atypical (a research parameter) by the analyzer. A previous study from Japan reported a high ability of the analyzer to predict the results of microscopic analysis of atypical cells in patients with urinary tract tumors; however, the type of the cancer cells is not specifically identified (5).

In this retrospective pilot study, we aimed to evaluate the application of an automated urine analyzer (AUA) for the diagnosis of $\mathrm{BC}$.

\section{Materials and Methods}

A retrospective data analysis of 2365 urine samples from the department of urology has been performed. We selected the samples of patients, who underwent cystoscopic evaluation or surgical treatment for various urological pathologies during 2018. Clinical records of these patients were further evaluated in order to select patients with recent or previous BC diagnosis. Out of 2365 urine samples, a total of 98 samples (one sample per patient) were evaluated within the preoperative two weeks before the cystoscopy in patients with BC diagnosis. A total of eight samples were selected from patients who underwent cystoscopic evaluation for non-cancerous etiologies (control group).

To assess the diagnostic efficacy of AUA, patients were divided into five groups according to the patient history of $B C$ risk groups, which are defined by the international guidelines $(6,7)$ :

Group 1-follow-up cystoscopy low-risk non-muscle invasive BC (NMIBC)

Group 2- follow-up cystoscopy high-risk NMIBC with previous intravesical Bacillus Calmette-Guerin (BCG) treatment (induction and at least 1-year maintenance treatment)

Group 3- transurethral resection for a newly diagnosed bladder mass without previous BC history

Group 4- radical cystectomy for muscle-invasive BC

Group 5- cystoscopic evaluation for non-cancerous etiologies (control group)

Epithelial cells, predominantly with high nucleic acid contents for their cell size, are counted as atypical (a research parameter) by the analyzer, and these cells in the atypical cell zone are reported as "atypical cell in $\mu \mathrm{L}$ " of the urine specimen.

\section{Statistical Analysis}

Data analysis was executed with SPSS version 20.0 (Chicago, IL, USA), and for the purpose of this pilot study, only the sensitivity and specificity analyses were performed.

\section{Results}

A total of 106 patients were included in this study, and the majority $(69.8 \%)$ of the patients were follow-up patients with previous diagnosis and treatment of NMIBC. For patients with low-risk NMIBC $(n=27)$, sensitivity and specificity were calculated as $75 \%$ and $100 \%$. Results of the urine analyses, cystoscopic evaluation, and pathologic characterization of the tumors are present in Table 1.
For patients with high-risk NMIBC $(n=47)$, who were previously treated with intravesical BCG, the sensitivity and specificity were calculated as $54.5 \%$ and $83.3 \%$, respectively. Results of the urine analyses, cystoscopic evaluation, and pathologic characterization of the tumors are present in Table 2.

For Group three patients $(n=17)$, who underwent transurethral resection for a newly diagnosed bladder mass and without previous BC history, the sensitivity and specificity were calculated as $50 \%$ and $100 \%$, respectively. Results of the urine analyses, cystoscopic evaluation, and pathologic characterization of the tumors are present in Table 3.

All patients in the radical cystectomy group $(n=7)$ with muscleinvasive $B C$ had positive urine analysis results for atypical cells. Furthermore, none of the patients in the control group $(n=8)$ had positive AUA results, and cystoscopic evaluation also did not show any bladder mass suspicious for BC.

\section{Discussion}

The results of this study showed acceptable sensitivity and specificity rates of the "fluorescence flow cytometry" based AUA compared to the current FDA approved urine tests. The results of the low-risk group are especially valuable regarding its potential use to decide on performing a follow-up cystoscopy or not. Such an application might be useful for avoiding an invasive procedure on the patient side together with additional economic savings considering the costs of the follow-up cystoscopies.

\begin{tabular}{|c|c|c|c|c|}
\hline \multirow{2}{*}{ SYSMEX result } & \multicolumn{3}{|c|}{ Cystoscopy result } & \multirow[b]{2}{*}{ Total } \\
\hline & Negative & LMPUN & $p T a L G$ & \\
\hline Negative & 23 & 0 & 1 & 24 \\
\hline Positive & 0 & 2 & 1 & 3 \\
\hline Total & 23 & 2 & 2 & 27 \\
\hline
\end{tabular}

NMIBC: Non-muscle invasive bladder cancer, LMPUN: Papillary urothelial neoplasia with low malignant potential

\begin{tabular}{|c|c|c|c|c|c|c|c|}
\hline \multirow{2}{*}{$\begin{array}{l}\text { SYSMEX } \\
\text { Result }\end{array}$} & \multicolumn{7}{|c|}{ Cystoscopy result } \\
\hline & Negative & LMPUN & pTaLG & pTaHG & pT1HG & MIBC & Total \\
\hline Negative & 30 & 3 & 1 & 1 & 0 & 0 & 35 \\
\hline Positive & 6 & 1 & 2 & 0 & 2 & 1 & 12 \\
\hline Total & 36 & 4 & 3 & 1 & 2 & 1 & 47 \\
\hline
\end{tabular}

\begin{tabular}{l|l|l|l|l|l|l|l|}
\hline \begin{tabular}{l} 
Table 3. First time TUR for a newly diagnosed bladder tumor \\
\multirow{2}{*}{$\begin{array}{l}\text { SYSMEX } \\
\text { result }\end{array}$}
\end{tabular} & \multicolumn{2}{l}{ Cystoscopy result } \\
\cline { 2 - 9 } & Negative & LMPUN & $p$ TaLG & $p$ TaHG & $p$ T1HG & MIBC & Total \\
\hline Negative & 1 & 1 & 4 & 1 & 1 & 1 & 9 \\
\hline Positive & 0 & 0 & 1 & 1 & 5 & 1 & 8 \\
\hline Total & 1 & 1 & 5 & 2 & 6 & 2 & 17 \\
\hline
\end{tabular}

TUR: Transurethral resection, MIBC: Muscle invasive bladder cancer, LMPUN: Papillary urothelial neoplasia with low malignant potential 
There are limited reports of the atypical cell counts with AUA for the diagnosis of BC. Ergüder et al. (8) analyzed 12900 urine samples retrospectively and included 1060 samples with the atypical parameter $>0.1$ in their study. The mean "atypical cell" count was significantly higher in patients with BC compared to other pathologies (1.61 vs 0.31 ). However, they did not report the pathological details of the tumors.

Shukuya et al. (5) analyzed the urine of 44 patients, who likely to have urinary tract tumors. In 41 patients, atypical cells were detected, and urinary tract tumors diagnosed. They reported the sensitivity as $81.0 \%$ and specificity as $88.0 \%$, however no data regarding the pathological details of the tumors. Our data showed sensitivity and specificity as $75 \%$ and $100 \%$ for patients with low-risk NMIBC. This group of patients is of interest to active surveillance, and an alternative approach with such a urine analyzer can contribute to the current efforts (9).

Almost half (44.3\%) of our study group included high-risk NMIBC patients who were treated with intravesical BCG. This group received at least 1-year maintenance treatment, which is the minimum recommended duration for intravesical treatment for high-risk patients. Cytology is useful, particularly as an adjunct to cystoscopy, in patients with high-risk tumors, and the sensitivity and specificity of the urine cytology were reported ranging from $30 \%$ and $86 \%$ to $83 \%$ and $43 \%$ in various studies, respectively (7). For this group, the sensitivity and specificity of the urine analyzer were calculated as $54.5 \%$ and $83.3 \%$.

\section{Study Limitations}

The present study is limited by its retrospective nature. A significant limitation is that we did not have another parameter such as urine cytology in all patients to compare with urine analyzer. Another limitation is the absence of information regarding the timing of the collection of the urine. According to the developers of the analyzer, the ideal urine should last at least 4 hours within the bladder or, most ideally, the first urine in the morning. Another potential limitation is that our study included a relatively small number of patients. However, compared to the limited reports, our data includes a relatively heterogeneous BC patient population that consisted of patients with different disease stages.

\section{Conclusions}

The results of this retrospective pilot study showed acceptable sensitivity and specificity rates of the "fluorescence flow cytometry" based AUA, and the results of the low-risk group are especially valuable regarding its potential use to decide on performing a follow-up cystoscopy or not. A prospective study is currently on progress to validate the findings of the current study.

\section{Acknowledgements}

Publication: This study was presented in the 14th International Urooncology Congress on November 6-10 in 2019 in Antalya Turkey.

Contribution: There is not any other contributors who may not be listed as authors.

Conflict of Interest: No conflict of interest was declared by the authors.

Financial Disclosure: The authors declared that this study received no financial support.

\section{Ethics}

Ethics Committee Approval: Retrospective study.

Informed Consent: Retrospective study.

Peer-review: Externally and internally peer-reviewed.

\section{Authorship Contributions}

Concept: İ.T., Design: İ.T., B.Ş., Ö.Y., Data Collection or Processing: S.S., R.A., D.F., Analysis or Interpretation: R.A., A.I.., I.T., D.F., M.B.A., Literature Search: S.S., Writing: I.T., H.K.Ç.

\section{References}

1. Bray F, Ferlay J, Soerjomataram I, Siegel RL, Torre LA, Jemal A. Global cancer statistics 2018: GLOBOCAN estimates of incidence and mortality worldwide for 36 cancers in 185 countries. CA Cancer J Clin 2018;68:394-424.

2. Bladder cancer: diagnosis and management of bladder cancer. BJU Int 2017;120:755-765.

3. Tan WS, Tan WP, Tan MY, et al. Novel urinary biomarkers for the detection of bladder cancer: A systematic review. Cancer Treat Rev 2018;69:39-52.

4. https://www.sysmex-europe.com/products/uf-50004000-3816. html

5. Shukuya K. 2016 Sysmex Customer Conference. Nagoya Japan. https:// www.science.gov/ topicpages/ffacial+dysmorphism+ spondylocarpal\#

6. https://www.auanet.org/guidelines/ bladder-cancer-non-muscle-invasive-guideline

7. https://uroweb.org/guideline/non-muscle-invasive-bladder-cancer/

8. Ergüder BI, Kücükkepeci EE, Mutlu M, Ardıç IN, Yiğit MS, Uruç MD, Ayhan Z, Ayaş MR. SB-24 IDRARDA "ATIPIK HÜCRE" PARAMETRESININ MESANE KANSERI VE DIĞER PATOLOJILER ILE ILIŞKISI. Uluslararası Laboratuvar Tıbbı ve XIX. Ulusal Klinik Biyokimya Kongresi. http:// tkb.dergisi.org/pdf/pdf_TKB_348.pdf

9. Hurle R. et al. Active surveillance for non-muscle invasive bladder cancer (NMIBC): Result from bladder cancer Italian active surveillance (BIAS) project. Eur Urol suppl. 2017:16:e1137-e1138. 